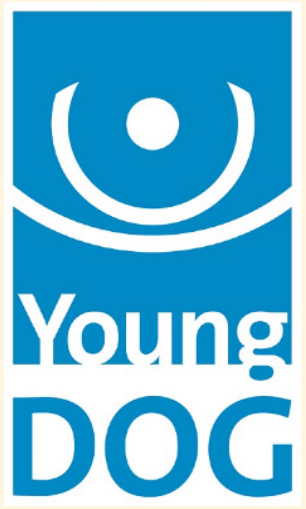

Die AG Young DOG richtet sich an junge Assistenzärzte und Nachwuchswissenschaftler. Sie fokussiert ihre Aktivitäten auf die Förderung des wissenschaftlichen Nachwuchses in der Ophthalmologie und die Vereinbarkeit von Klinik, Forschung und Familie.

Die Mitgliedschaft in der AG ist den Mitgliedern der DOG vorbehalten. Mitglied werden können Assistenzärzte, habilitierte Ärzte und Wissenschaftler sowie Professoren bis W 2. Nähere Information zur AG Young DOG finden Sie unter http://www. dog.org/?cat=137.

\section{Bettina Hohberger}

Augenklinik, Universitätsklinikum Erlangen, Erlangen, Deutschland

\section{Korrespondenzadresse:}

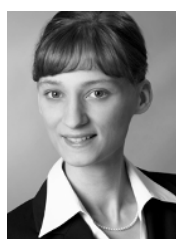

Dr. med. Dr. rer. biol. hum Bettina Hohberger Augenklinik, Universitätsklinikum Erlangen

Schwabachanlage 6, 91054 Erlangen, Deutschland

Bettina.hohberger@uk-erlangen.de

Ophthalmologe $2021 \cdot 118: 78-80$ https://doi.org/10.1007/s00347-02001270-8

(c) Springer Medizin Verlag $\mathrm{GmbH}$, ein Teil von Springer Nature 2020

\title{
Die „DOG 2020 online“ - erstmals im von-Graefe-Jahr
}

Das Jahr 2020 wird sicherlich in die Geschichtsbücher eingehen. Wer hätte Anfang dieses Jahres daran gedacht, dass ein Virus innerhalb kürzester Zeit das öffentliche Leben, Beruf und Privatleben beeinflussen wird. Die Corona-Pandemie hat vor keinem Bereich gestoppt. Eine Herausforderung stellte die Umstellung vieler Veranstaltungen und Tagungen auf ein virtuelles Format dar. So entschied sich auch die DOG, ihren Kongress nicht abzusagen, sondern virtuell durchzuführen. Erstmals seit ihrem Bestehen fand vom 9. bis 11. Oktober die „DOG 2020 online“ statt. Wir haben für Sie Nachwuchsärzte/ -forscher sowie langjährige Kliniker und Wissenschaftler zu ihrem Eindruck zur virtuellen DOG befragt. Zudem interviewten wir die Preisträgerin des diesjährigen von-Graefe-Preises und ehemalige Mentorin der Young DOG.

\section{„Hat der virtuelle Charakter} der DOG einen Einfluss auf die Präsentation der Beiträge?"

Dr. T. Stahnke (Wissenschaftler, Rostock): Ich stand der diesjährigen DOG im Vorfeld eher skeptisch gegenüber, da ich bisher keinerlei Erfahrungen mit einer „Online-Tagung“ sammeln konnte. Zum Start der DOG habe ich mich schnell in die sehr anwenderfreundliche Gestaltung der virtuellen „Räume“ und in den Ablauf der einzelnen Sessions hineingefunden. Ich war positiv überrascht über den Ablauf der Sitzungen, meine Skepsis war verflogen, und ich habe viele interessante Beiträge verfolgt, welche anschließend diskutiert werden konnten. Einer meiner eigenen Beiträge wurde bereits am ersten Tag in einer freien Beitragssit- zung präsentiert. Auch wenn der persönliche Charakter durch die Präsentationsform fehlte, konnte man fast keine Unterschiede im Vergleich zu einer Präsenzveranstaltung feststellen. Weiterhin hatte ich den Eindruck, dass der virtuelle Charakter für die Diskussionen sogar von Vorteil war, da aus einer zum Teil bestehenden Anonymität heraus auch Fragen gestellt wurden, welche auf einem realen Kongress mit Präsenzbeiträgen gar nicht gestellt worden wären.

\section{„Gewinnt die Forschung durch den virtuellen Charakter eine} andere Bedeutung?"

Dr. S. Reinehr (Wissenschaftlerin, Bochum): Die Forschung selbst lebt durch einen regen Austausch an Ideen. Neue Ansätze und Konzepte entwickeln sich oft einfach, indem man durch die Postersession läuft oder sich spontan in einen Vortrag setzt. Dadurch erhält man oft neue oder überraschende Denkanstöße. Auch der Austausch mit Kollegen und Kooperationspartnern ist ein wichtiger Part einer Konferenz. All dies kann bei guten Angeboten auch virtuell stattfinden, ist aber durchaus schwieriger. Ein wichtiger Aspekt für die Forschung ist der Schutz der Daten. Auf Konferenzen werden oft Daten vorgestellt, die so noch nicht veröffentlicht wurden. Wenn nicht sichergestellt werden kann, dass diese Daten ungehindert zugängig sind, ist es für Forschende schwieriger, diese zu präsentieren. Dies kann dazu führen, dass noch nicht veröffentlichte Daten nur noch intern besprochen werden und so kaum noch neuer Input von außen kommt. Doch vermutlich braucht man nur Zeit, sich an die neue Situation zu gewöhnen. Die Forschung wird immer wichtig sein und auch neue Wege durch die Krise finden.

„Sie kennen das virtuelle Format bereits von Ihrem Studium - wie haben Sie als Referent den Kongress erlebt?" A. Dittert (Student, Erlangen): Unser Studium musste sich innerhalb weniger Wochen infolge der Corona-Pandemie in seinem digitalen Angebot um ein nie geahntes Ausmaß erweitern und hat dies bis auf ein paar Startschwierigkeiten gut gelöst. So war es für mich keine Überraschung, dass die DOG 2020 online stattfinden muss. Während des Kongresses gefiel mir die innovative Wahl der Benutzeroberfläche besonders. Ich hatte im Vorfeld ehrlicherweise damit gerechnet, dass man sich relativ simpel in Listen die jeweiligen Vortrags-Headlines raussucht und darauf klickt. Aber die frische Kacheloptik im „Home“ gab dem Ganzen eine gute Einteilung. Für mich als erstmaligen Referenten war es auf der einen Seite schade, nicht die Erfahrung eines LiveVortrags mitgenommen zu haben. Auf der anderen Seite, machte die zwanglose Kommunikation mit dem Team der DOG es sehr einfach, den Vortrag digital und zur eigenen Zufriedenheit zur Verfügung zu stellen.

„Sie haben das Consilium diagnosticum mitverfolgt - was waren Ihre Eindrücke?"

L. Hübner (Assistenzärztin, Erlangen): Aufgrund der großen Anzahl an Referenten bot das Consilium diagnosticum eine technische Herausforderung für Veranstalter und Vortragende. Traditionell werden in diesem 
Symposium 16 ungewöhnliche Kasuistiken in kurzen Beiträgen präsentiert. Dies sollte auch in der diesjährigen, etwas anderen Auflage des Kongresses nicht ausbleiben. Ein großes Lob gilt in diesem Jahr dem Technikteam, welches die Zusammenschaltung aller Referenten betreute und bei technischen Schwierigkeiten mit Lösungsansätzen zur Seite stand. Schließlich ist es gelungen, durch das Abspielen der im Vorfeld angefertigten Beiträge mit den sich anschließenden Live-Diskussionen den uns bekannten, mit dem einen oder anderen Schmankerl behafteten Schlussbeitrag zum Freitagabend zu gestalten.

\section{„Brachte das neue Format der diesjährigen DOG eine beson- dere Herausforderung?"}

Prof. T. Fuchsluger (Direktor, Rostock): Das Online-Format der DOG im Jahre 2020 war im Vergleich zu anderen OnlineTagungen beeindruckend aufwendig. Trotz einer Vielzahl von wissenschaftlichen Sitzungen, Firmensymposien und Updates funktionierte der Ablauf im Wesentlichen reibungslos. Durch das spätere Abrufen der Inhalte erhält die DOG 2020 zusätzliche Attraktivität. Hoffen wir auf baldige Präsenztreffen zur Wiederaufnahme persönlicher Kontakte.

\section{„Als amtierende Obfrau der Julius Hirschberg Gesellschaft hatten Sie vor, Ihren Jahreskon- gress in enger Verbindung mit dem der DOG durchzuführen, um die Feierlichkeiten zum} 150. Todestag von Albrecht von Graefe gemeinsam zu begehen, oder?"

Dr. S. Scholtz (Obfrau der Julius Hirschberg Gesellschaft, Heidelberg): Ja, der Vorstand der Julius Hirschberg Gesellschaft hatte beschlossen, anlässlich der Ehrung von Graefes ihren Kongress in enger Anbindung und Abstimmung mit Prof. Hoerauf und der DOG zeitgleich im Estrel durchzuführen. Wir hatten die komplette Planung für den JHGKongress schon im Februar abgeschlossen. Als COVID-19 die DOG zum Kurswechsel zwang, war ich total begeistert, dass die DOG den Sprung ins kalte Wasser eines Online-Kongresses wagte und nicht einfach wie viele andere ihren Kongress absagte. Für mich war die DOG ein großes Vorbild: Dieser Mut, in so kurzer Zeit die Kongresswelt neu zu erfinden, hat mich motiviert, auch unseren Hirschberg-Kongress online zu gestalten - was uns beiden, der großen und der kleinen Gesellschaft, sehr gut gelungen ist!

\section{„Der von-Graefe-Preis im von-Graefe-Jahr - eine ganz besondere Auszeichnung. Was für einen Ratschlag können Sie unseren jungen Leserinnen und Lesern mit auf den Weg geben, die gerade dabei sind, ihren Weg im Bereich der ophthal- mologischen Forschung zu beginnen?"}

Prof. U. Schlötzer-Schrehardt (Wissenschaflerin, Erlangen): Über die Verleihung des vonGraefe-Preises habe ich mich als Naturwissenschaftlerin gleich doppelt gefreut, denn dadurch wird unsere wissenschaftliche Arbeit in ganz besonderer Weise gewürdigt. Dies verdeutlicht auch einmal mehr den Auftrag der DOG, ophthalmologische Grundlagenforschung und klinische Forschung gleichermaßen zu fördern, und bestätigt mich in meinem gewählten Karriereweg, der natürlich nicht nur mit solchen Höhepunkten, sondern immer wieder auch mit Rückschlägen verbunden war. Ein großer Vorteil von Anfang an war jedoch die enge Verzahnung zwischen unseren Forschungseinrichtungen mit dem klinischen Umfeld, die den direkten Zugang zu klinisch relevanten Fragestellungen ermöglicht und mein Interesse an der Aufklärung von Krankheitsmechanismen zur Entwicklung neuer Therapieansätze nachhaltig geprägt hat. Diese Zusammenhänge haben sich seit den Zeiten Albrecht von Graefes nicht wesentlich verändert, was durch ein Zitat seines Schülers Theodor Leber, entnommen aus der Biografie zum 150. Todestag: Albrecht von Grafe (1828-1870) von Prof. Jens Martin Rohrbach, anschaulich belegt wird: „Von ihm (A.v. Graefe) habe ich gelernt, wie klinische Erfahrungen, pathologischanatomische Beobachtungen und experimentelle Ergebnisse sich gegenseitig ergänzen und durchdringen müssen, damit wir zur Einsicht in verwickelte Vorgänge gelangen."

Wer sich wie ich als Basic Scientist für eine Laufbahn in der medizinischen Grundlagenforschung entscheidet, ergreift einen der interessantesten Berufe überhaupt, der es ermöglicht, zeitlebens neue Erkenntnisse zu gewinnen und diese an den wissenschaftlichen Nachwuchs, an Kliniker und an Patienten weiterzugeben. Gleichzeitig bringt diese Entscheidung aber ein hohes Maß an Risikobereitschaft mit sich, da die prekäre Stellensituation an den Universitäten, insbesondere im akademischen Mittelbau, bekanntermaßen kaum Planungssicherheit zulässt. Für Frauen kommt erschwerend hinzu, dass die Zeit der Qualifizierungsphase mit der Familienplanung zusammenfällt. In dieser Phase müssen sie sich unter hohem Konkurrenzdruck gegenüber ihren männlichen Mitbewerbern im Kampf um die wenigen Professorenstellen durch Publikationsleistung und Drittmitteleinwerbung durchsetzen. Trotz dieser Erschwernisse kenne ich viele hervorragende Wissenschaftlerinnen in der experimentellen und klinischen Augenforschung, die als motivierende Rollenvorbilder und Mentorinnen fungieren können. Ihre
Karrierewege können beispielhaft aufzeigen, wie man mit Leidenschaft, Risikobereitschaft, Flexibilität und einer gehörigen Portion Durchhalte- und Durchsetzungsvermögen eine wissenschaftliche Laufbahn bei gleichzeitiger Berücksichtigung familiärer Wünsche einschlagen kann. Neue Regelungen und Empfehlungen, wie beispielsweise die Anerkennung familienbedingter Auszeiten bei Bewerbungsverfahren durch Bewertung des „akademischen Alters", versuchen hierbei Hilfestellung zu leisten. 
Hier steht eine Anzeige.

\section{Springer}

